\section{Stable non-Gaussian noise parameter modulation in digital communication}

\section{M.E. Çek and F.A. Savaci}

The parameter of the stable non-Gaussian noise sequence is modulated by the binary message sequence to achieve a secure communication system. The characteristic exponent 'alpha' of a stable non-Gaussian noise sequence carries the binary information. The receiver of the proposed random communication system demodulates the received signal by estimating the parameters of the transmitted noise sequence to recover the binary message sequence.

Introduction: Spread spectrum techniques, by embedding the narrow band message signal into a wide band carrier signal, ensure secure communication. As an alternative to the conventional digital communication systems, chaos-based digital communication techniques became popular by the beginning of the 1990s and chaotic signals have since been a proper candidate to ensure the security due to their broadband nature [1-6]. Chaotic signals have been used to encode the binary message bits with chaos shift keying techniques [4, 5]. Since most of the power of a broadband chaotic signal is at the low frequencies, to perform security in communication might not be possible, as explained in [7]. Owing to the limited security performance of chaotic signals novel techniques are needed. The idea of adding white noise to hide the information combined with chaotic signals to achieve security was proposed in [8].

In this Letter, the new proposed technique employs the symmetric $\alpha$-stable distributed noise ( $\mathrm{S} \alpha \mathrm{S}$ ) which is modulated by the message signal. This new technique can be called 'stable non-Gaussian noise parameter modulation' since the binary message is encoded by the parameters of the $\alpha$-stable noise sequence. Although the idea of using a noise-like carrier goes back to the 1950s $[6,9]$, to the best of our knowledge in the literature only this newly introduced technique uses such a random sequence for encoding the message signal instead of using deterministic signals. Such a random sequence for encoding the message signal can then be considered as a random carrier. In the sequel, first, brief information about the $\alpha$-stable distribution is given and then the estimation of the parameters in the receiver is presented based on the method introduced in [10].

Alpha-stable distribution: The random variable $X \sim S_{\alpha}(\gamma, \beta, \mu)$ is said to have $\alpha$-stable distribution if the characteristic exponent associated with $X$ is given as

$$
\phi(\theta)= \begin{cases}\exp \left\{j \mu \theta-\gamma^{\alpha}|\theta|^{\alpha}\left(1-j \beta \operatorname{sign}(\theta) \tan \left(\frac{\alpha \pi}{2}\right)\right)\right\} & \text { if } \alpha \neq 1 \\ \exp \left\{j \mu \theta-\gamma|\theta|\left(1+j \beta \frac{2}{\pi} \operatorname{sign}(\theta) \ln |\theta|\right)\right\} & \text { if } \alpha=1\end{cases}
$$

with the parameters $0<\alpha \leq 2,-1 \leq \beta \leq 1, \gamma \geq 0$ and $\mu \in(-\infty, \infty)$ [11].

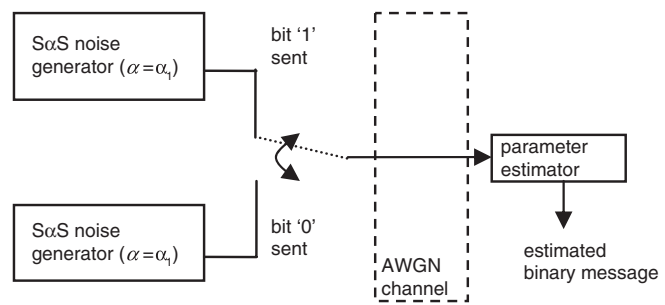

Fig. 1 Block diagram of proposed random communication scheme

Transmitter structure: In the proposed communication scheme, shown in Fig. 1, the binary message is encoded by the parameter $\alpha$ of the symmetric $\alpha$-stable noise (i.e. $\beta=\mu=0$ ) and then the corresponding noise sequence is transmitted through the channel. If the message bit is logic ' 1 ' (respectively ' 0 ') then $\alpha_{1}$-stable (respectively $\alpha_{2}$-stable) noise sequence is transmitted. During the bit length $T_{b}$ the samples $x_{k}$ are drawn from a probability density ' $S_{\alpha_{i}}(1,0,0)$ ' $i=1$, (respectively $i=$
2) if $m\left(n T_{b}\right)=1$ (respectively $\left.m\left(n T_{b}\right)=0\right)$, i.e.

$$
x_{k} \sim \begin{cases}S_{\alpha_{1}}(1,0,0) & \text { if } m\left(n T_{b}\right)=1 \\ S_{\alpha_{2}}(1,0,0) & \text { if } m\left(n T_{b}\right)=0\end{cases}
$$

where $k=n T_{b}+1, \ldots, n T_{b}+T_{b}$ and $n=0,1, \ldots$

The transmitted noise sequence is shown in Fig. 2 together with the combined arbitrary bit stream with $T_{b}=10^{4}$ and $\alpha_{1}=1, \alpha_{2}=0.5$. The $\alpha$-stable distributed noise sequence $x_{k}$ has been generated by the method given in [12].
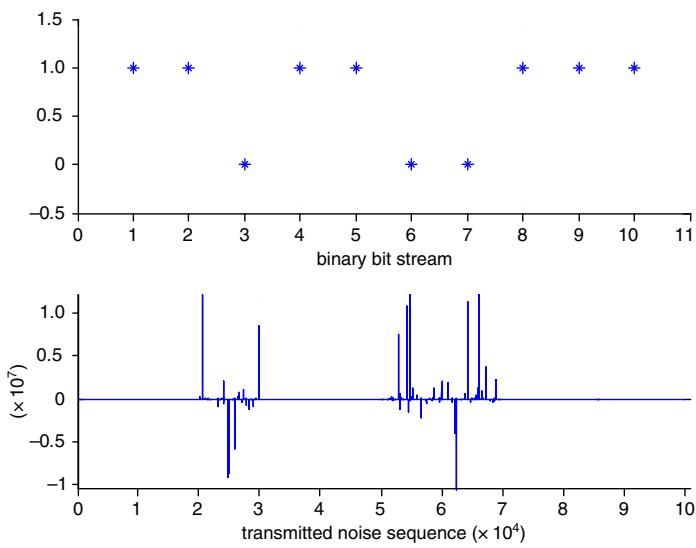

Fig. 2 Message bit stream (upper), transmitted signal in time domain (lower)

Receiver structure (estimating the characteristic exponent): The receiver of the proposed communication scheme estimates the characteristic exponent of the received noise sequence and a hard limiter determines the sent binary message. The estimation of the characteristic exponent has been performed by the sinc estimator introduced in [10] which computes the characteristic exponent $\alpha$ of the noise sequence $x(k)$, $k=1,2, \ldots, T_{b}$ from the following equation:

$$
\sin c\left(\frac{p \pi}{\alpha}\right)=\left[\frac{p \pi}{2}\left(\frac{A_{p} A_{-p}}{\tan p \pi / 2}\right)+S_{p} S_{-p} \tan \frac{p \pi}{2}\right]^{-1}
$$

where $p$ is the fractional moment order, $T_{b}$ is the number of samples for each bit and the fractional moments $A_{p}$ and $S_{p}$ are given as

$$
A_{p}=\frac{1}{N} \sum_{k=1}^{T_{b}}|x(k)|^{p}, \quad S_{p}=\frac{1}{N} \sum_{k=1}^{T_{b}} \operatorname{sgn}(x(k))|x(k)|^{p}
$$

The bit stream with the corresponding actual and estimated values of the alpha parameter at the receiver, without noise at the channel, is shown in Fig. 3.
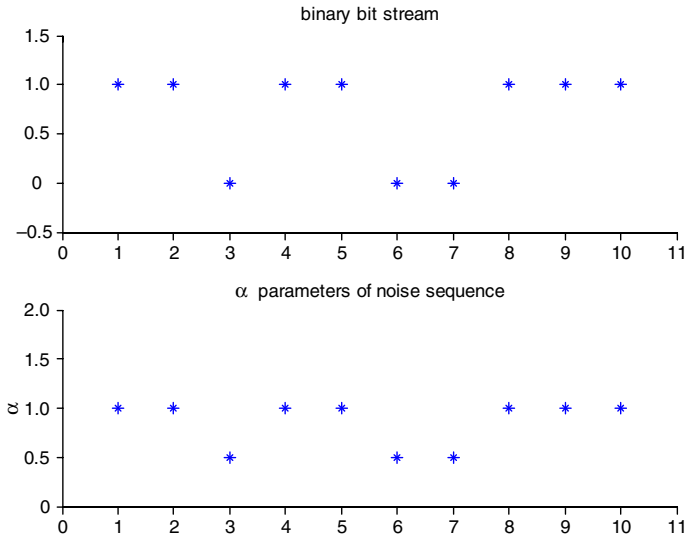

Fig. 3 Message bit stream (upper). For each message bit, estimated alpha parameter values from $S \alpha S$ noise sequence (no noise in channel) (lower)

Results: The impulsiveness parameter 'alpha' can be properly estimated at the receiver if the length of the generated noise sequence is properly chosen and from these estimated alpha values the binary message can be decoded. Although the effect of the additive white Gaussian noise (AWGN) channel has been observed as small deviations in the actual alpha values, by properly choosing the threshold in the detector the 
same amount of deviations occur in the alpha values and hence the actual alpha values can still be estimated as shown in Fig. 4 in case the dispersion parameter $\gamma$ of $\mathrm{S} \alpha \mathrm{S}$ is equal to the dispersion parameter of the AWGN channel.
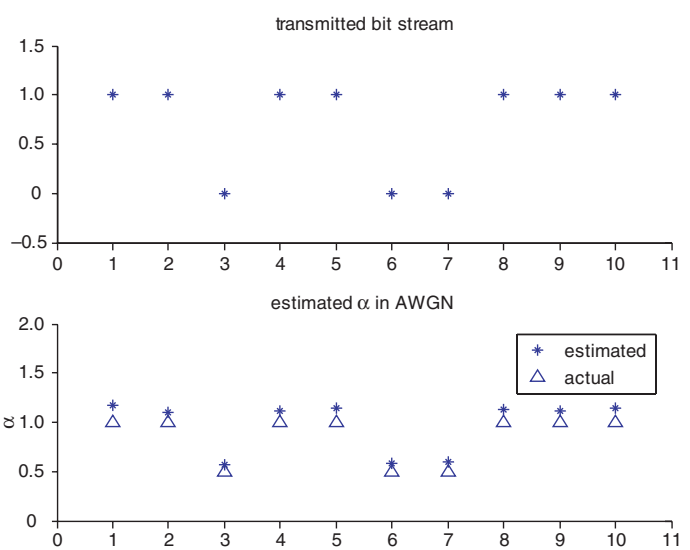

Fig. 4 Message bit stream (upper). For each message bit, estimated and actual alpha parameter values from $S \alpha S$ noise sequence (AWGN in channel) (lower)

Conclusion: The proposed communication scheme can be considered as a random communication system since the $\mathrm{S} \alpha \mathrm{S}$ noise sequence for encoding acts as a random carrier. The bit error rate (BER) performance of the proposed method under the AWGN channel will be investigated as a further work.

C) The Institution of Engineering and Technology 2009

6 August 2009

doi: $10.1049 / \mathrm{el} .2009 .2280$

M.E. Çek and F.A. Savaci (Department of Electrical and Electronic Engineering, Izmir Institute of Technology, Urla 35430, Izmir, Turkey)

E-mail: emrecek@iyte.edu.tr

\section{References}

1 Abel, A., and Schwarz, W.: 'Chaos communications - principles, schemes, and system analysis', Proc. IEEE, 2002, 90, (5), pp. 691-710

2 Cuomo, K.M., Oppenheim, A.V., and Strogatz, H.: 'Synchronization of Lorenz based chaotic circuits with applications to communications', IEEE Trans. Circuits Syst., 1993, 40, (10), pp. 626-633

3 Lau, F.C.M., and Tse, C.K.: 'Chaos-based digital communication systems' (Springer, 2003)

4 Stavroulakis, P.: 'Chaos applications in telecommunication' (Taylor \& Francis, 2006)

5 Schimming, T., and Hasler, M.: 'Optimal detection of differential chaos shift keying', Int. J. Bifurcation Chaos, 2000, 47, (11), pp. 1712-1719

6 Kolumban, G., Lau, F.C.M., and Tse, C.K.: 'Generalization of waveform communications: the Fourier analyzer approach', Circuits Syst. Signal Process., 2005, 24, (5), pp. 451-474

7 Savaci, F.A., Yalçin, M.E., and Güzeliş, C.: 'Steady-state analysis of nonlinearly coupled Chua's circuit with periodic input', Int. J. Bifurcation Chaos, 2003, 13, (11), pp. 3395-3407

8 Zhang, B., Chen, M., Zhou, D., and Li, Z.: 'Particle-filter-based estimation and prediction of chaotic states', Chaos Solitons Fractals, 2007, 32, pp. 1491-1498

9 Basore, B.L.: 'Noise-like signals and their detection by correlation', $\mathrm{Ph}$. D. Thesis, MIT, Cambridge, MA, 1952

10 Kuruoğlu, E.E.: 'Density parameter estimation of skewed alphastable distributions', IEEE Trans. Signal Process., 2001, 49, (10), pp. 2192-2201

11 Samorodnitsky, G., and Taqqu, M.S.: 'Stable non-gaussian random processes' (Chapman \& Hall/CRC, 1994)

12 Janicki, A., and Weron, A.: 'Simulation and chaotic behaviour of $\alpha$-stable stochastic processes' (Marcel Dekker, 1994) 